\title{
Prospective analysis of bedside percutaneous subdural tapping for the treatment of chronic subdural haematoma in adults
}

\author{
Marcus H T Reinges, Isabell Hasselberg, Veit Rohde, Wilhelm Küker, Joachim M Gilsbach
}

\begin{abstract}
Objectives-Although there is general agreement that surgery is the best treatment for chronic subdural haematoma (CSDH), the extent of the surgical intervention is not well defined.

Methods-The less invasive surgical technique of bedside percutaneous subdural tapping and spontaneous haematoma efflux after twist drill craniostomy under local anaesthesia was prospectively analysed in 118 adult patients, 99 with unilateral and 19 with bilateral CSDH.

Results-The mean number of subdural tappings was 3.2. Ninety two of the patients with unilateral CSDH were successfully treated by up to five subdural tappings, $95 \%$ of the patients with bilateral CSDH were successfully treated by up to 10 subdural tappings. The mean duration of inpatient treatment was 12 days. In 11 patients $(9 \%)$ the treatment protocol had to be abandoned because of two acute subdural bleedings, two subdural empyemas, and seven cases of insufficient haematoma efflux and no neurological improvement. The only significant predictor for failure of the described treatment protocol was septation visible on preoperative CT.

Conclusions-The described therapy protocol is-apart from a purely conservative treatment-the least invasive presently available surgical technique for treating chronic subdural haematoma. Its results are comparable with other modern treatment protocols. Thus, it can be recommended in all patients as a first and minimally invasive therapy, especially in patients in a poor general condition. Patients with septation visible on preoperative CT should be excluded from this form of treatment.

(F Neurol Neurosurg Psychiatry 2000;69:40-47)
\end{abstract}

Keywords: chronic subdural haematoma; percutaneous subdural tapping; twist drill trephination

Chronic subdural haematoma $(\mathrm{CSDH})$ is one of the most frequent types of intracranial haemorrhage and carries a favourable prognosis when treated adequately. However, the optimal treatment is not yet defined. Even though there are reports about successful nonsurgical treatment, ${ }^{1-4}$ to date there is general agreement that surgery is the best therapy. ${ }^{5-25-31}$ The most often used surgical treatment is that of burr hole craniostomy with subsequent continuous closed catheter drainage with or without irrigation. ${ }^{5} 69-12161719-2123262931$ As to the extent of drainage, some authors advocate surgical removal of the CSDH as completely as possible. ${ }^{52}$ By contrast, other study groups have reported that complete evacuation of the haematoma seems to be unnecessary. ${ }^{912} 1920$ Thus, the question arises, if there is really any need for continuous and extensive haematoma drainage, or continuous irrigation of the subdural space, or both, or if a less invasive surgical alternative exists.

The purpose of this prospective study was to analyse our experiences of bedside subdural tapping and spontaneous haematoma efflux without irrigation or continuous drainage after twist drill craniostomy under local anaesthesia, and to assess the advantages and shortcomings of this procedure. To our knowledge, no prospective study on the efficacy of this treatment protocol-which is similar to the method already reported ${ }^{7822}$-has hitherto been published.

Patients and methods

Between 1995 and 1998, 122 consecutive patients with chronic subdural haematoma were treated in our institution. Patients with an acute subdural haematoma-that is, patients presenting with symptoms within three days of trauma - and patients with subdural hygroma containing xanthochromic or thin bloody fluid were excluded from the evaluation. Four patients were treated by a different therapy regimen and were, therefore, also excluded. The remaining group of 118 patients consisted of 71 men $(60 \%)$ and 47 women $(40 \%)$, ranging in age from 19 to 95 years (mean 67 years).

To facilitate comparison with previously reported studies, patients were assessed with the most commonly used neurological grading system for $\mathrm{CSDH}$ devised by Markwalder et $a l^{19}$ as follows:

Grade 0: patient neurologically normal

Grade 1: patient alert and oriented; mild symptoms, such as headache; absent or mild symptoms or neurological deficit, such as reflex asymmetry

Grade 2: patient drowsy or disoriented with variable neurological deficit, such as hemiparesis

Grade 3: patient stuporous but responding appropriately to noxious stimuli; severe focal signs, such as hemiplegia

Grade 4: patient comatose with absent motor response to painful stimuli; decerebrate or decorticate posturing.

The initial diagnosis was confirmed by CT, and treatment was initiated if the thickness of the haematoma exceeded that of the skull and 
A

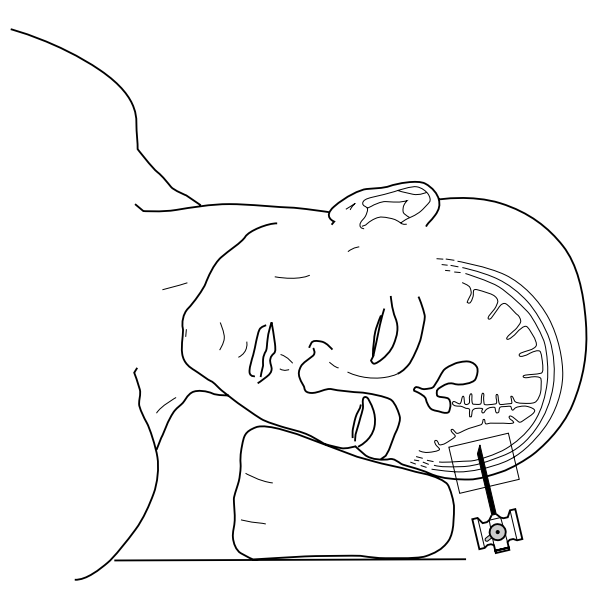

B

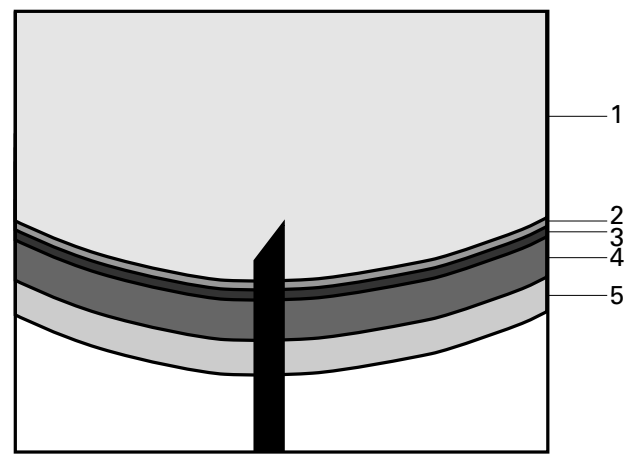

Figure 1 Positioning of the head for evacuation of chronic subdural haematoma by spontaneous efflux. (A) Overview. (B) Partial magnification. The patient is placed in the $30^{\circ}$ Trendelenburg position and the head is placed such that the teflon cannula is situated at the deepest point of the haematoma cavity. To prevent injury to the brain the cannula is introduced so that it exceeds the tabula interna of the skull only slightly. 1 =haematoma cavity; $2=$ outer neomembrane; $3=$ dura; $4=$ scull; $5=$ skin.

if the patient was symptomatic - that is, at least grade 1 .

All patients were treated at the bedside by percutaneous subdural tapping under local anaesthesia. After partial shaving and disinfection, a stab incision of the skin was performed at the site of maximum thickness of the CSDH, preferably anterior to the coronal suture. Twist drill trephination was performed using a $3 \mathrm{~mm}$ hand driven drill. After perpendicular penetration of the skull, the dura and the outer neomembrane of the haematoma were perforated with a needle. Thereafter, a $14 \mathrm{G}$ teflon cannula was perpendicularly introduced, exceeding the tabula interna of the skull only slightly. The haematoma was evacuated only by spontaneous efflux and Valsalva's manoeuvre with the patient positioned in a $30^{\circ}$ Trendelenburg position with the head placed so that the cannula was situated at the deepest point of the haematoma cavity (fig 1). After cessation of $\mathrm{CSDH}$ efflux, the cannula was removed and the skin was closed by a single suture with the patient still in the $30^{\circ}$ Trendelenburg position. The amount of haematoma efflux was quantified and immediately postoperatively the patient was examined clinically. A control by CT was performed within the next 48 hours. If the

Table 1 Neurological grade according to the grading system of Markwalder et al ${ }^{19}$ preoperatively, after the last subdural tapping, and at follow up evaluation mean 22 (range 1-46) months postoperatively

\begin{tabular}{|c|c|c|c|c|c|c|}
\hline \multirow[b]{2}{*}{ Neurological grade } & \multicolumn{2}{|c|}{ Preoperatively } & \multicolumn{2}{|c|}{$\begin{array}{l}\text { After last subdural } \\
\text { tapping }\end{array}$} & \multicolumn{2}{|c|}{$\begin{array}{l}\text { At follow up } \\
\text { evaluation } \star / t\end{array}$} \\
\hline & $n$ & $(\%)$ & $n$ & $(\%)$ & $n$ & $(\%)$ \\
\hline 0 & 0 & 0 & 89 & 75 & 86 & 80 \\
\hline 1 & 23 & 19 & 28 & 24 & 7 & 6 \\
\hline 2 & 79 & 67 & 0 & 0 & 0 & 0 \\
\hline 3 & 13 & 11 & 0 & 0 & 0 & 0 \\
\hline 4 & 3 & 3 & 0 & 0 & 0 & 0 \\
\hline Dead $\ddagger$ & & & 1 & 1 & 15 & 14 \\
\hline
\end{tabular}

*Including seven and two patients who were finally operated on using continuous drainage after burr hole trephination and/or membranectomy after craniotomy because of insufficient haematoma evacuation and subdural empyema, respectively, and two patients who were operated on acutely via craniotomy and haematoma evacuation because of procedure related acute subdural bleeding.

†10 patients were lost for long term follow up.

$\ddagger$ In all patients death was related to concomitant diseases and not to the treatment or to a recurrence of $\mathrm{CSDH}$ thickness of the residual haematoma still exceeded that of the skull and if the patient was still symptomatic, the procedure was repeated. If there was no septation visible on CT, the repeated tapping was performed through the same burr hole. If there was septation visible or if the amount of haematoma efflux was below $10 \mathrm{ml}$, repeated tapping was performed through a new burr hole. If repeated subdural tappings did not improve neurological grade to at least grade 1, secondary treatment by continuous drainage after burr hole trephination and/or membranectomy after craniotomy was initiated. The patients were discharged the day after the last subdural tapping if their signs and symptoms were reduced at least to grade 1 .

After discharge, the patients were controlled clinically and by CT examination within 6 weeks, and whenever there was clinical suspicion of haematoma reaccumulation. An indication for readmission to hospital and reoperation was made if the patients showed typical signs and symptoms and reaccumulation of the haematoma exceeding the thickness of the skull. Before completion of this study, in all patients the referring physicians were asked for a re-evaluation.

\section{Results}

Table 1 shows the patients of the present series, classified according to their preoperative neurological grade secondary to the grading system by Markwalder et al. ${ }^{19}$

The aetiology of CSDH and the distribution of concomitant diseases are shown in table 2 . In the group of patients with known head trauma in the past, the mean duration from trauma to therapy was 6 weeks, ranging from 0.5 to 20 weeks.

The CSDH was right sided in 40 patients $(34 \%)$, left sided in 59 patients $(50 \%)$, and bilateral in 19 patients (16\%) (fig 2). Septation was visible on the first CT in 25 patients $(21 \%)$ (fig 3). On initial CT the mean thickness of the CSDH was $2 \mathrm{~cm}$, ranging from 1 to $3.5 \mathrm{~cm}$. The density of the CSDH on first CT is shown in table 3. 
Table 2 Aetiology of CSDH and concomitant diseases in 118 patients

\begin{tabular}{lcc}
\hline & $n$ & $(\%)$ \\
\hline Aetiology: & 72 & 61 \\
$\quad$ Trauma & 46 & 39 \\
Unknown & & \\
Concomitant diseases: & 45 & 38 \\
Cardiovascular & 43 & 36 \\
Arterial hypertension & 35 & 30 \\
Neurological & 29 & 25 \\
Anticoagulation therapy & 20 & 17 \\
Diabetes mellitus & 11 & 9 \\
Malignant tumour & 11 & 9 \\
Pulmonary & 8 & 7 \\
Alcohol misuse & & \\
\hline
\end{tabular}

${ }^{\star}$ More than one disease per patient is possible.

The number of subdural tappings for each patient is shown in fig 4 . The mean number of subdural tappings in the 99 patients with unilateral $\mathrm{CSDH}$ was 2.8 , ranging from 1 to 10; the mean number in the 19 patients with bilateral $\mathrm{CSDH}$ was 5.0, ranging from two to 11. Altogether, the mean number of subdural
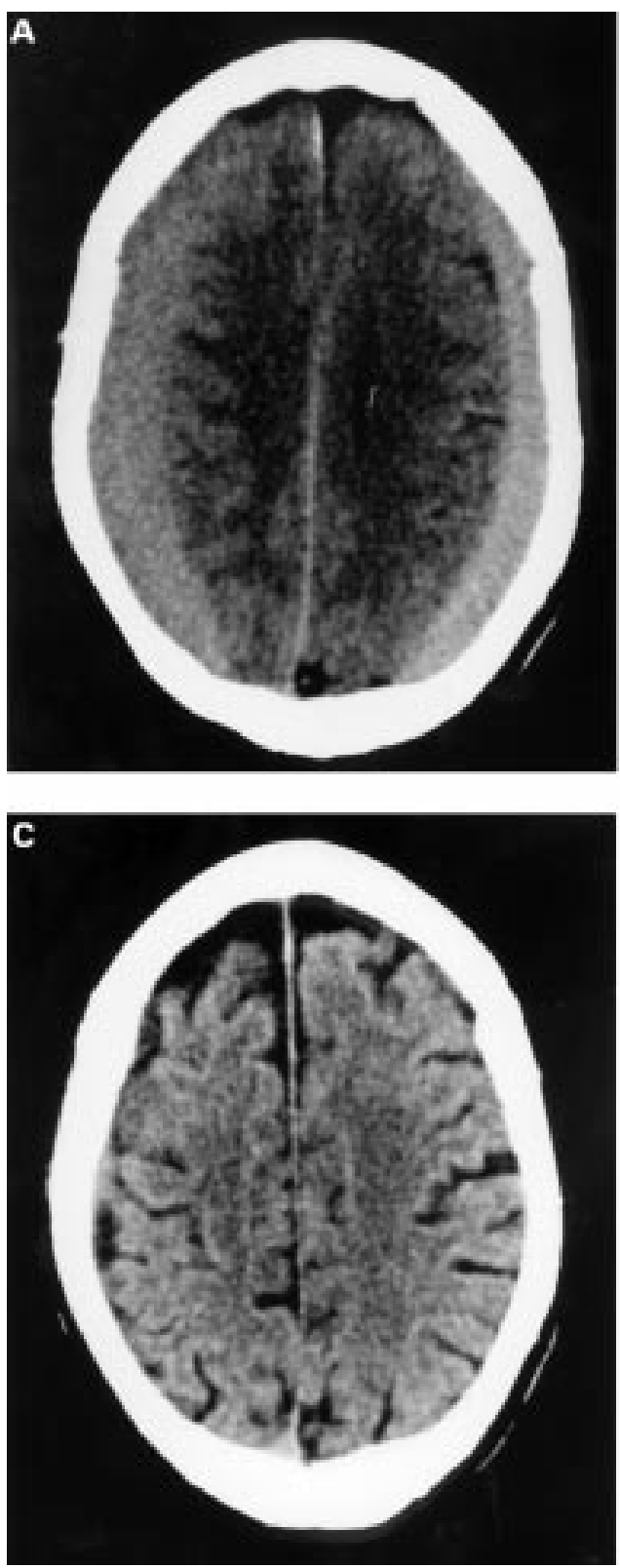

tappings in all 118 patients was 3.3. Ninety one out of the 99 patients with unilateral $\mathrm{CSDH}$ $(92 \%)$ were treated by up to five tappings and 18 out of the 19 patients with bilateral CSDH $(95 \%)$ were treated by up to 10 subdural tappings. Altogether, 377 subdural tappings through 225 twist drill craniostomies were performed. In 66 out of the 377 procedures (18\%) no or only up to $10 \mathrm{ml}$ haematoma efflux was achieved. Immediately after every subdural tapping the neurological status ameliorated in 253 procedures $(67 \%)$, worsened in two procedures $(1 \%)$, and remained stable in 115 procedures $(31 \%)$.

In five out of the 118 patients (4\%)-that is, in five out of 377 subdural tappings (1.3\%)acute subdural bleeding and in one patient $(1 \%)$ intracerebral bleeding was seen on early postoperative CT. No correlation between these haemorrhagic complications and the surgeon's experience could be demonstrated. Two patients $(2 \%)$ had to be operated on acutely

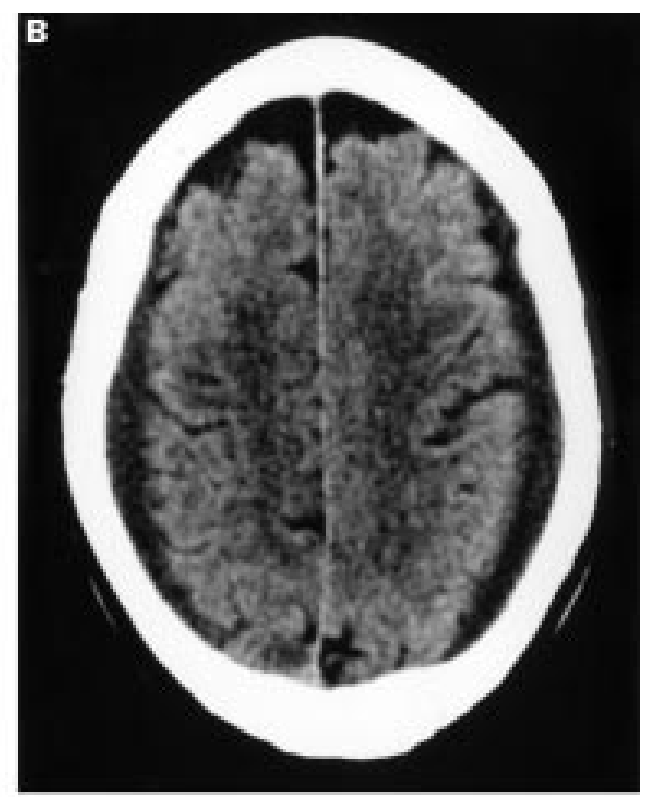

Figure 2 Serial CT of a 66 year old man with bilateral chronic subdural haematoma who presented with left sided hemiparesis and headache. (A) Scan obtained before treatment, demonstrating bilateral hyperdense haematoma. (B) Scan obtained 1 month after first subdural

tapping, - that is, after three right sided and three left sided subdural tappings, demonstrating reduction of the - now hypodense - haematoma. After the sixth subdural tapping the patient was free of signs and symptoms. (C) Scan obtained at follow up 4 months after the last subdural tapping, demonstrating complete re-expansion of the brain. 

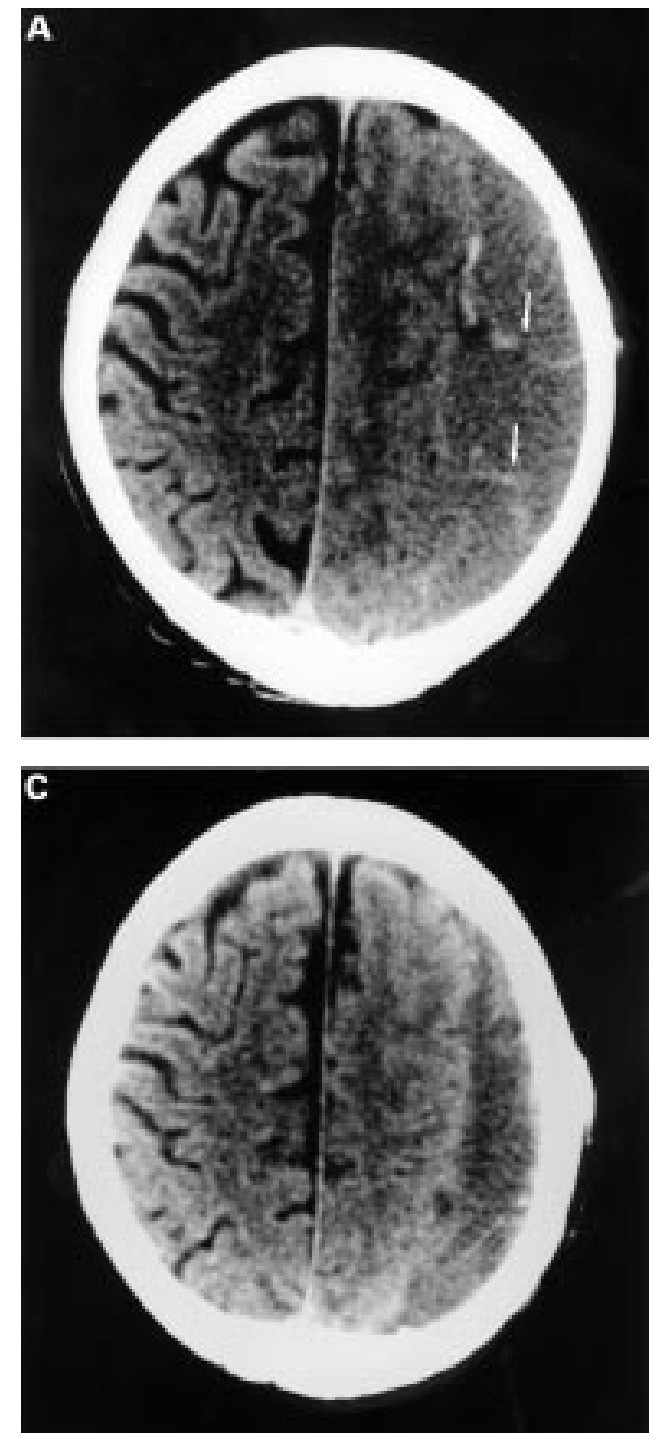

Table 3 Density of CSDH on first CT of 137 CSDH in 118 patients (99 unilateral and 19 bilateral CSDH)

\begin{tabular}{lcl}
\hline Density & $n$ & $(\%)$ \\
\hline Hypodense & 52 & 38 \\
Hypodense-isodense & 13 & 10 \\
Hypodense-hyperdense & 29 & 21 \\
Isodense & 29 & 21 \\
Isodense-hyperdense & 2 & 1 \\
Hyperdense & 12 & 9 \\
\hline
\end{tabular}

due to a procedure related acute subdural bleeding with acute worsening of the neurological status. Seven patients $(6 \%)$ were finally operated on using continuous drainage after burr hole trephination and/or membranectomy after craniotomy because of insufficient haematoma evacuation and failure to improve in neurological status (fig 3). Subdural empyema occurred in three patients (3\%) after five, six, and 10 subdural tappings, resulting in operative revision in two patients $(2 \%)$. Thus 11 patients in total $(9 \%)$ were finally treated by a different protocol (table 4).

Symptomatic postoperative pneumocephalus was not seen in any patient. No patient died as a consequence of the operative procedure,

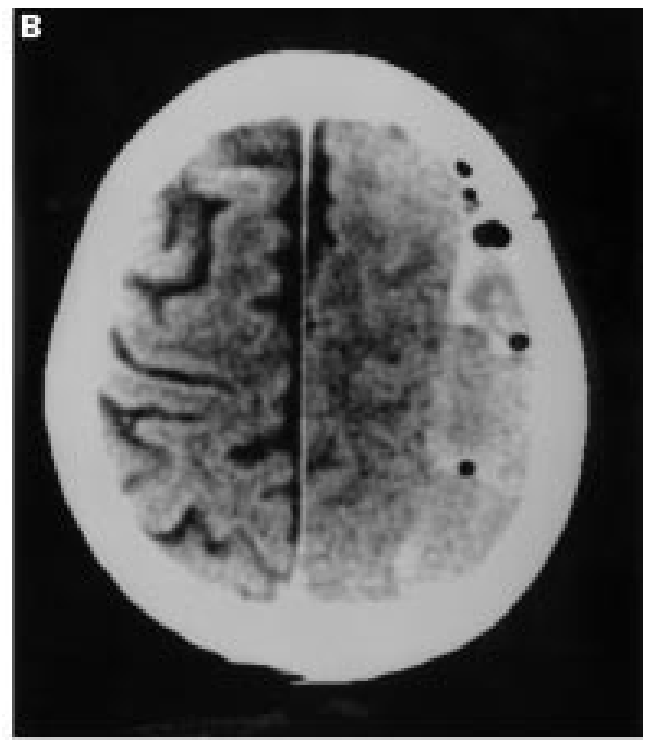

Figure 3 Serial CT of an 83 year old man with a left sided chronic subdural haematoma who presented with right sided hemiparesis and headache. The patient was treated by eight subdural tappings without sufficient haematoma evacuation and without permanent improvement of neurological status. Therefore, he was finally treated by craniotomy, evacuation of the haematoma, and continuous catheter drainage. (A) Scan obtained before treatment, showing septation of the isodense haematoma (arrows). (B) Scan obtained 1 day after first subdural tapping through a left frontal twist drill craniostomy, demonstrating slight reduction of haematoma size and postoperative intracranial air. Note air bubbles at the septa. (C) Scan obtained after eight subdural tappings (17 days after first subdural tapping), still demonstrating the - now hypodense-

haematoma, although it is somewhat reduced in size.

one patient $(1 \%)$ died during her hospital stay from stroke due to cardiogenic embolism.

The mean duration of inpatient treatment was 12 days with a range from 1 to 35 days. Apart from the one patient $(1 \%)$ who died, $75 \%$ of the patients were free of signs and symptoms after the last subdural tapping, the remaining $24 \%$ presented with only mild signs and symptoms - that is, grade 1 according to the grading system of Markwalder et al. ${ }^{19}$ Postoperative neurological grading after the last subdural tapping and at the time of follow up evaluation, mean 22 (range 1-46) months after the last subdural tapping is shown in table 1. Fifteen patients $(13 \%)$ died within the follow up period. However, death was related in all cases to concomitant diseases such as heart failure or malignant tumour and not to a recurrence of CSDH.

Statistical analysis showed a highly significant correlation $\left(\chi^{2}\right.$ 18.556; Fisher's exact test, $\mathrm{p}<0.001$ ) between septation visible on preoperative CT and patients who had finally to be treated by a changed treatment protocol because of insufficient haematoma evacuation. No other significant predisposing factors for unsuccessful treatment were found. 


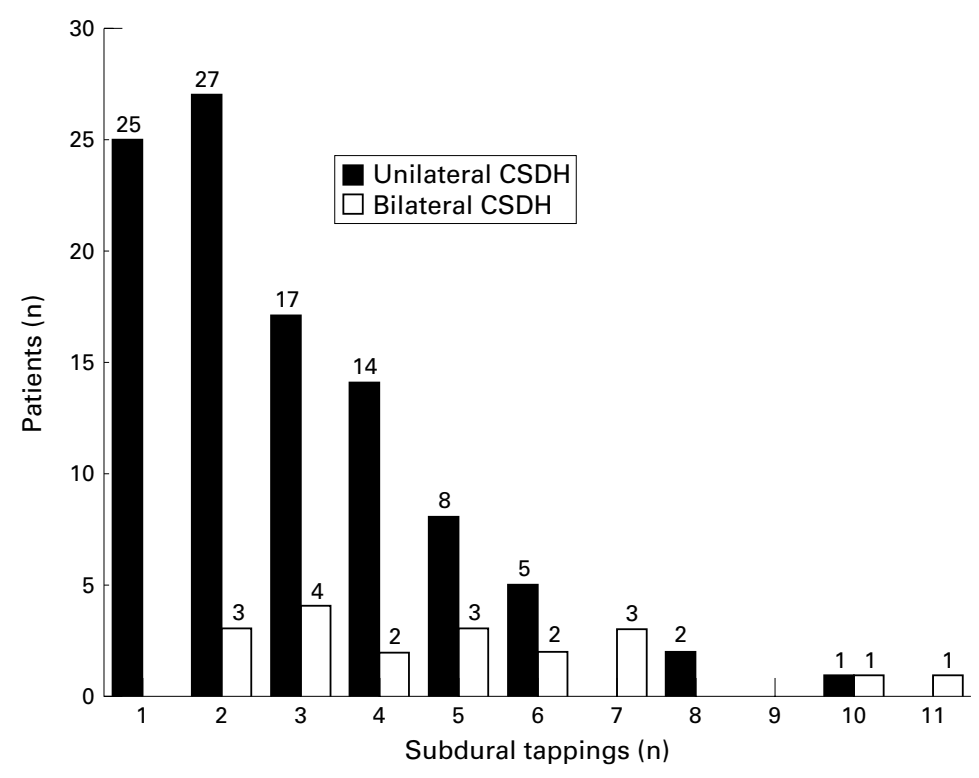

Figure 4 Number of treated patients (99 patients with unilateral and 19 patients with bilateral CSDH) in relation to the number of subdural tappings in each patient. The figure includes two patients with unilateral CSDH, acutely operated on via craniotomy and haematoma evacuation because of procedure related acute subdural bleeding after the first subdural tapping. Further, two patients with unilateral CSDH are included, operated on via craniotomy and evacuation of empyema and CSDH. Finally, seven patients are included, operated on via continuous drainage and/or membranectomy because of insufficient haematoma evacuation after 1, 2, 3, 4, 4, 8, and 8 subdural tappings, (see also table 4).

\section{Discussion}

Even though there is general agreement that the treatment of CSDH should be surgical, the extent of surgical therapy is still under debate. ${ }^{5-31}$ However, it has been demonstrated that increased surgical invasiveness does not guarantee better results. ${ }^{1428}{ }^{29}$ For example, the worst surgical results were reported after extensive craniotomy and membranectomy. ${ }^{28} 29$

The current theory about persistence, enlargement, and healing of CSDH is that there is local hyperfibrinolysis with a large concentration of fibrinolytic enzymes in the outer neomembrane and in the subdural fluid. ${ }^{33-36}$ Further, endothelial gap junctions of macrocapillaries in the outer neomembrane potentially lead to leakage of blood substances into the membrane and the haematoma cavity. ${ }^{37-39}$ Finally, it is the ratio between rebleeding and reabsorption of the subdural collection which determines whether the $\mathrm{CSDH}$ will resolve, persist, or increase. ${ }^{32}{ }^{36}$ The improvement of local hyperfibrinolysis by reducing the amount of fibrinolytic enzymes through surgical removal of the CSDH leads to a tightening of the outer neomembrane and to a decrease in repetitive haemorrhage from the macrocapillaries, in the case of healing resulting in progressive increase of collagen and concurrent reduction of macrocapillaries. ${ }^{32} 38$

Thus Aoki and Drapkin advocated surgical removal of CSDH as completely as possible. ${ }^{52}$ By contrast, other authors reported that complete drainage of the haematoma seemed to be unnecessary. ${ }^{9}{ }^{12} 1920$ The probable pathophysiological basis is that incomplete haematoma evacuation may change the ratio between rebleeding and reabsorption towards reabsorption and, thus initiate a self healing process.

Consequently, there have been reports on various surgical attempts to treat $\mathrm{CSDH}$ radically on the one hand, but as minimally invasive as possible on the other hand. In 1968, Burton reported the management of CSDH by twist drill craniostomy and aspiration of the subdural haematoma. ${ }^{8}$ In 1975 , Negrón et al reported on seven patients with subdural haematoma, five of whom had been successfully treated by bedside needle trephination. ${ }^{22}$ Hubschmann reported 22 cases of $\mathrm{CSDH}$

Table 4 Synopsis of patients of the present series which were finally treated by a changed treatment protocol

\begin{tabular}{|c|c|c|c|c|c|c|c|c|c|}
\hline Patient & $\begin{array}{l}\text { Age } \\
(y)\end{array}$ & Sex & $\begin{array}{l}\text { Preop } \\
\text { neurol } \\
\text { grade }^{\star}\end{array}$ & $\begin{array}{l}\text { Thickness } \\
\text { of } \\
\text { CSDH } \\
(\mathrm{cm})\end{array}$ & $\begin{array}{l}\text { Septation } \\
\text { visible }\end{array}$ & $\begin{array}{l}\text { Number } \\
\text { of } \\
\text { subdural } \\
\text { tappings }\end{array}$ & $\begin{array}{l}\text { Number } \\
\text { of } \\
\text { subdural } \\
\text { tappings } \\
\text { with up to } \\
10 \mathrm{ml} \\
\text { haematoma } \\
\text { efflux }\end{array}$ & Reason to change the treatment protocol & Final treatment \\
\hline 1 & 61 & M & 2 & 1.0 & No & 1 & 0 & $\begin{array}{l}\text { Acute subdural bleeding with } \\
\text { deterioration of neurological grade }\end{array}$ & $\begin{array}{l}\text { Craniotomy, evacuation of acute bleeding and of } \\
\text { CSDH }\end{array}$ \\
\hline 2 & 70 & $\mathrm{~F}$ & 2 & 3.0 & No & 1 & 0 & $\begin{array}{l}\text { Acute subdural bleeding with } \\
\text { deterioration of neurological grade }\end{array}$ & $\begin{array}{l}\text { Craniotomy, evacuation of acute bleeding and of } \\
\text { CSDH }\end{array}$ \\
\hline 3 & 74 & M & 2 & 2.5 & Yes & 10 & 3 & Subdural empyema & $\begin{array}{l}\text { Craniotomy, evacuation of CSDH and of } \\
\text { empyema, continuous catheter drainage }\end{array}$ \\
\hline 4 & 64 & M & 3 & 3.5 & Yes & 6 & 3 & Subdural empyema & $\begin{array}{l}\text { Craniotomy, evacuation of CSDH and of } \\
\text { empyema, continuous catheter drainage }\end{array}$ \\
\hline 5 & 66 & $M$ & 4 & 2.5 & No & 1 & 0 & $\begin{array}{l}\text { Poor preoperative neurological grade, } \\
\text { no improvement of neurological } \\
\text { grade }\end{array}$ & $\begin{array}{l}\text { Burr hole trephination and continuous catheter } \\
\text { drainage }\end{array}$ \\
\hline 6 & 63 & $\mathrm{~F}$ & 2 & 2.0 & Yes & 4 & 3 & $\begin{array}{l}\text { Insufficient evacuation of CSDH, no } \\
\text { improvement of neurological grade }\end{array}$ & $\begin{array}{l}\text { Craniotomy, evacuation of CSDH, partial } \\
\text { membranectomy }\end{array}$ \\
\hline 7 & 64 & $\mathrm{M}$ & 2 & 2.5 & Yes & 3 & 3 & $\begin{array}{l}\text { Insufficient evacuation of CSDH, no } \\
\text { improvement of neurological grade }\end{array}$ & $\begin{array}{l}\text { Burr hole trephination and continuous catheter } \\
\text { drainage }\end{array}$ \\
\hline 8 & 47 & $\mathrm{~F}$ & 2 & 2.5 & Yes & 4 & 3 & $\begin{array}{l}\text { Insufficient evacuation of CSDH, no } \\
\text { improvement of neurological grade }\end{array}$ & $\begin{array}{l}\text { Craniotomy, partial membranectomy, continuous } \\
\text { catheter drainage }\end{array}$ \\
\hline $9 \dagger$ & 83 & $M$ & 2 & 3.0 & Yes & 8 & 3 & $\begin{array}{l}\text { Insufficient evacuation of CSDH, no } \\
\text { improvement of neurological grade }\end{array}$ & $\begin{array}{l}\text { Craniotomy, evacuation of CSDH, continuous } \\
\text { catheter drainage }\end{array}$ \\
\hline 10 & 72 & $M$ & 2 & 2.5 & Yes & 2 & 0 & $\begin{array}{l}\text { Insufficient evacuation of CSDH, } \\
\text { seizure after subdural tapping }\end{array}$ & $\begin{array}{l}\text { Burr hole trephination and continuous catheter } \\
\text { drainage }\end{array}$ \\
\hline 11 & 75 & $\mathrm{M}$ & 2 & 3.0 & Yes & 8 & 4 & $\begin{array}{l}\text { Insufficient evacuation of CSDH, no } \\
\text { improvement of neurological grade }\end{array}$ & $\begin{array}{l}\text { Burr hole trephination and continuous catheter } \\
\text { drainage }\end{array}$ \\
\hline
\end{tabular}

${ }^{\star}$ According to the grading system by Markwalder $e t \mathrm{al}^{19}$

†See also figure 3

$\mathrm{CSDH}=$ chronic subdural haematoma. 
treated by twist drill craniostomy and closed system drainage. ${ }^{17}$ In his series, one patient required repeated twist drill evacuation of the haematoma, another patient required craniotomy to achieve total haematoma removal, and in two patients the procedure failed. ${ }^{17}$ Aoki presented a study of 24 patients with $\mathrm{CSDH}$ treated by subdural tapping and spontaneous evacuation of the haematoma. ${ }^{5}$ However, because of a recurrence rate of $29 \%$ he changed to subdural tapping and irrigation, resulting in a recurrence rate of $7 \% .^{5}$ Finally, Burchiel and Taylor treated 131 patients by serial twist drill craniostomy and haematoma aspiration. ${ }^{7}$ In their study, $73 \%$ of the patients achieved significant neurological improvement, 23\% required an additional operative procedure. ${ }^{7}$

The therapy protocol used here is similar to that already described. ${ }^{57822}$ However, to our knowledge, no prospective study on the efficacy of this form of treatment has been documented.

The epidemiological data of the present series are similar to other published series dealing with the treatment of $\mathrm{CSDH},{ }^{1-17}{ }^{19-32} 40$ so that the results are comparable. To evaluate the described therapy regimen according to the duration from trauma to therapy, we subsumed $\mathrm{CSDH}$ and so called subacute subdural haematoma - that is, haematoma more than 3 weeks old and haematoma between 3 days and 3 weeks old. ${ }^{40}$

In accordance with other authors, ${ }^{9} 121920$ we found that it is unnecessary to remove the subdural collection completely. A partial reduction of the CSDH may suffice to change the imbalance of rebleeding and reabsorption towards reabsorption and, thus, healing, may be due to an improvement of local hyperfibrinolysis or due to a reduction of tensile forces on the macrocapillaries. However, a considerable reduction in volume is necessary to initiate this self healing process, as Ito et al have shown that between 6 and 24 hours after infusion of labelled erythrocytes, haemorrhage into the haematoma cavity accounted for a mean of $6.7 \%$ of the haematoma content. ${ }^{34}$ Correspondingly, in the present series an indication for reoperation was made if the remaining or reaccumulating haematoma exceeded the thickness of the skull and the patient was symptomatic.

The rationale for treating CSDH by bedside percutaneous tapping after twist drill craniostomy under local anaesthesia without continuous drainage or irrigation is that it offers a less invasive treatment alternative to other presently available surgical treatment protocols. In the treatment protocols with drainage by placing the subdural drain obliquely and deeply into the haematoma cavity through one or more burr holes, there is always the danger of creating acute subdural bleeding or of damaging the brain by uncontrolled placement of the drain. ${ }^{262731}$ Further, by placing a drain into the haematoma cavity and by irrigating the subdural space, there is the risk of not only damaging superficial cerebral vessels or bridging veins, but also of damaging macrocapillaries of the outer neomembrane of the $\mathrm{CSDH}$, potentially resulting in acute subdural bleeding or reaccumulation. ${ }^{2627}{ }^{31}$ There is also the risk of damage to fragile vessels and the neomembrane by creating negative subdural pressure due to evacuation of the CSDH by suction and even by gravity forces in continuous subdural drains. Thus, in the present series evacuation of the CSDH was achieved only by spontaneous efflux and Valsalva's manoeuvre with the patient placed in the $30^{\circ}$ Trendelenburg position. Further, continuous catheter drainage and irrigation carry the risk of infection. ${ }^{20} 232627{ }^{31}$ Wound infection or meningitis occurred in $18 \%$ of the patients reported by Smely et al. ${ }^{26}$ We tried to reduce the risk of infection by immediate removal of the subdural cannula after cessation of spontaneous efflux of the haematoma in every subdural tapping. Finally, in all surgical techniques for treating $\mathrm{CSDH}$ there is the risk of postoperative pneumocephalus, potentially resulting in increased intracranial pressure. ${ }^{6234142}$ The risk of pneumocephalus is reduced by the described treatment protocol, as the skin is closed immediately after cessation of spontaneous blood efflux with the patient still in the $30^{\circ}$ Trendelenburg position.

However, here also the therapy regimen does not overcome some of the shortcomings and risks of conventional surgical therapy of $\mathrm{CSDH}$ :

By repeated subdural tapping after twist drill craniostomy there is the risk of damaging the cerebral surface with each tapping, resulting in five acute subdural bleedings and one intracerebral bleeding in our series, requiring acute craniotomy and haematoma evacuation in two patients (table 4). However, this rate is comparable with other series, which also reported procedure related acute bleedings. ${ }^{26}{ }^{27} 31$ This risk can be reduced by the use of a twist drill that does not penetrate the skull ${ }^{43} 44$ and by careful placement of the perpendicularly introduced teflon cannula only slightly exceeding the tabula interna of the skull.

By repeated subdural tapping, the risk of wound infection and subdural empyema increases, resulting in three subdural empyemas after 10, six, and five subdural tappings in the present series, requiring reoperation via craniotomy in two patients (table 4). However, this rate of wound infection and subdural empyema is similar to other published series, ranging between $1 \%$ and $18 \% .^{1214-162324262831}$ The risk of infection should be reducible by defining a maximal number of subdural tappings before changing to another therapy protocol, as in the present series in the two patients requiring craniotomy and evacuation of the subdural empyema, infection occurred after 10 and six subdural tappings for unilateral CSDH (table 4). Thus, in our opinion, the maximal number of subdural tappings should not exceed five in unilateral CSDH and 10 in bilateral $\mathrm{CSDH}$, as in the present series $92 \%$ and $95 \%$ of the patients, respectively, were successfully treated by five and 10 subdural tappings.

The risk of pneumocephalus is reduced by the treatment protocol, resulting in no symptomatic postoperative pneumocephalus in the 
present series. Therefore, subdural tapping should be performed in the $30^{\circ}$ Trendelenburg position with Valsalva's manoeuvre to prevent air from penetrating into the subdural space.

The $6 \%$ rate of patients who had to be treated finally by continuous drainage and/or membranectomy because of insufficient haematoma evacuation and failure to improve in neurological status is similar to the reported rate of reoperation in other series, ranging between $2 \%$ and $37 \%$ of recurrence and

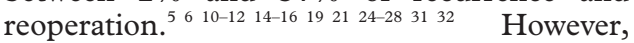
these patients can potentially be identified by extensive septation visible on preoperative CT before the procedure, as septation was the only significant predictor for unsuccessful treatment in the present series. There were no other significant correlations found, especially not between the need for changing the therapy regimen and the thickness of the $\mathrm{CSDH}$ on preoperative CT, by contrast with the report of Ram and colleagues and in accordance with Stoobandt and colleagues. ${ }^{23} 27$

The mean duration of inpatient treatment of 12 days in the present series is comparable with other recently reported surgical treatment regimens which range between 4.9 and 23 days of mean hospital stay. ${ }^{71112152124262932}$ However, it has to be considered that in the present series many patients were in a poor general condition, so called subacute subdural haematoma were included, and that 19 patients with bilateral CSDH required bilateral therapy. Using the described treatment protocol, the duration of hospital stay should also be reducible by defining the above mentioned maximal number of subdural tappings as five in unilateral and 10 in bilateral $\mathrm{CSDH}$, and to change to another therapeutic regimen after that number is reached without significant improvement of the neurological status. This may be the compromise between less invasiveness on the one hand and maximum effectiveness on the other.

Finally, the clinical outcome of our patients with (apart from one patient who had died during her hospital stay from stroke due to cardiogenic embolism) $75 \%$ of the patients being free of signs and symptoms and the remaining $24 \%$ of the patients presenting with only mild signs and symptoms after the last subdural tapping is at least as good as in other, more invasive, modern surgical therapy protocols. $^{69-12} 14-17192124-27293132$

\section{Conclusions}

The ideal surgical treatment of CSDH has not been definitely defined. However, in the present prospective series the less invasive surgical technique of bedside percutaneous subdural tapping and spontaneous haematoma efflux after twist drill craniostomy under local anaesthesia has resulted in satisfactory results. It can be performed on the ward with a complication rate as low as other modern surgical techniques. We recommend it in all patients as a first and minimally invasive attempt, especially in patients in poor general condition. Patients with extensive septation visible on preoperative CT should be excluded. If the procedure fails after five subdural tappings in unilateral CSDH and after 10 subdural tappings in bilateral CSDH (five subdural tappings on each side), the regimen should be changed to prevent a rise in surgical morbidity. However, secondary treatment by continuous drainage after burr hole trephination and/or membranectomy after craniotomy-which are much more invasive and carry a higher procedure related morbidity should be performed only if the described initial treatment protocol fails.

1 Bender MB, Christoff N. Non surgical treatment of subdural hematomas. Arch Neurol 1974;31:73-9.

2 Lusins J, Jaffe R, Bender MB. Unoperated subdural hematomas. Long-term follow-up study by brain scan and electroencephalography. $\mathcal{F}$ Neurosurg 1976;44:601-7.

3 Naganuma H, Fukamachi A, Kawakami M, et al. Spontaneous resolution of chronic subdural hematomas. Neurosurgery 1986;19:794-8.

4 Suzuki J, Takaku A. Nonsurgical treatment of chronic subdural hematoma. I Neurosurg 1970;33:548-53.

5 Aoki N. Subdural tapping and irrigation for the treatment of chronic subdural hematoma in adults. Neurosurgery 1984; 14:545-8.

6 Benzel EC, Bridges RM Jr, Hadden TA, et al. The single burr hole technique for the evacuation of non-acute subdural hematomas. F Trauma 1994;36:190-4

7 Burchiel and Taylor, cited by: Camel M, Grubb RL Jr. Treatment of chronic subdural hematoma by twist-drill craniostomy with continuous catheter drainage. $f$ Neurosurg 1986;65:183-7.

8 Burton C. The management of chronic subdural hematoma using a compact hand twist drill. Mil Med 1968;133:891-5.

9 Camel M, Grubb RL Jr. Treatment of chronic subdural hematoma by twist-drill craniostomy with continuous hematoma by twist-drill craniostomy with
catheter drainage. $\mathcal{F}$ Neurosurg $1986 ; 65: 183-7$.

10 Eggert HR, Harders A, Weigel K, et al. Relapses after burrhole drainage of chronic subdural haematomas. Neurochirurgia 1984;27:141-3.

11 Ernestus R-I, Beldzinski P, Lanfermann H, et al. Chronic subdural hematoma: surgical treatment and outcome in 104 patients. Surg Neurol 1997;48:220-5.

12 Gilsbach J, Eggert H-R, Harders A. External closed drainage treatment of chronic subdural hematomas after bore-hole trepanation. Unfallchirurgie 1980;6:183-6.

13 Gjerris F, Schmidt K. Chronic subdural hematoma. Surgery or mannitol treatment. $\mathcal{F}$ Neurosurg 1974;40:639-42.

14 Grisoli F, Graziani N, Peragut JC, et al. Perioperative lumbar injection of Ringer's lactate solution in chronic subdural hematomas: a series of 100 cases. Neurosurgery 1988;23: 616-21.

15 Hamilton MG, Frizzell JB, Tranmer BI. Chronic subdural hematoma: the role for craniotomy reevaluated. Neurosurgery 1993;33:67-72.

16 Harders A, Eggert HR, Weigel K. Treatment of chronic subdural haematoma by closed external drainage. Neurochirurgia 1982;25:147-52.

17 Hubschmann OR. Twist drill craniostomy in the treatment of chronic and subacute subdural hematomas in severly ill of chronic and subacute subdural hematomas in

18 Markwalder T-M. Chronic subdural hematomas: a review. $\mathcal{F}$ Neurosurg 1981;54:637-45.

19 Markwalder T-M, Steinsiepe KF, Rohner M, et al. The course of chronic subdural hematomas after burr-hole craniostomy and closed-system drainage. $\mathcal{F}$ Neurosurg 1981;55:390-6.

20 Markwalder TM, Seiler RW. Chronic subdural hematomas: to drain or not to drain? Neurosurgery $1985 ; 16: 185-8$.

21 Mellergard P, Wisten O. Operations and re-operations for chronic subdural haematomas during a 25 -year period in a well defined population. Acta Neurochir (Wien) 1996;138: 708-13.

22 Negrón RA, Tirado G, Zapater C. Simple bedside technique for evacuating chronic subdural hematomas. technique for evacuating chronic subdural

23 Ram Z, Hadani M, Sahar A, et al. Continuous irrigationdrainage of the subdural space for the treatment of chronic subdural haematoma. A prospective clinical trial. Acta Neurochir (Wien) 1993;120:40-3.

24 Robinson RG. Chronic subdural hematoma: surgical management in 133 patients. F Neurosurg 1984;61:263-8.

25 Sambasivan M. An overview of chronic subdural hematoma: experience with 2300 cases. Surg Neurol 1997; 47:418-22.

26 Smely C, Madlinger A, Scheremet R. Chronic subdural haematoma-a comparison of two different treatment modalities. Acta Neurochir (Wien) 1997;139:818-26.

27 Stroobandt G, Fransen P, Thauvoy C, et al. Pathogenetic factors in chronic subdural haematoma and causes of recurrence after drainage. Acta Neurochir (Wien) 1995;137: $6-14$.

28 Svien HJ, Gelety JE. On the surgical management of encapsulated subdural hematoma. A comparison of the results of 21:172-7 
29 Tabaddor K, Shulman K. Definitive treatment of chronic subdural hematoma by twist-drill craniostomy and closedsubdural hematoma by twist-drill craniostomy
system drainage. $\mathcal{F}$ Neurosurg $1977 ; 46: 220-6$.

30 Tyson G, Strachan E, Newman P, et al. The role of craniectomy in the treatment of chronic subdural hematomas. $\mathcal{F}$ Neurosurg 1980;52:776-81.

31 Weisse A, Berney J. Chronic subdural haematomas. Results of a closed drainage method in adults. Acta Neurochi (Wien) 1994;127:37-40.

32 Drapkin AJ. Chronic subdural hematoma: pathophysiological basis for treatment. Br F Neurosurg 1991;5:467-73.

33 Ito H, Komai T, Yamamoto S. Fibrinolytic enzyme in the lining walls of chronic subdural hematoma. $\mathcal{F}$ Neurosur 1978;48:197-200.

34 Ito H, Yamamoto S, Saito K, et al. Quantitative estimation of hemorrhage in chronic subdural hematoma using the ${ }^{51} \mathrm{Cr}$ erythrocyte labeling method. 7 Neurosurg 1987;66:862-4.

35 Ito $\mathrm{H}$, Saito K, Yamamoto S, et al. Tissue-type plasminogen activator in the chronic subdural hematoma. Surg Neurol 1988;30:175-9.

36 Labadie EL, Glover D. Local alterations of hemostaticfibrinolytic mechanisms in reforming subdural hematomas. Neurology 1975;25:669-75.

37 Sato S, Suzuki J Ultrastructural observations of the capsule of chronic subdural hematoma in various clinical stages. $\mathcal{F}$
Neurosurg 1975;43:569-78.

38 Yamashima T, Yamamoto S, Friede RL. The role of endothelial gap junctions in the enlargement of chronic subdural hematomas. F Neurosurg 1983;59:298-303.

39 Yamashima T, Yamamoto S. How do vessels proliferate in the capsule of a chronic subdural hematoma? Neurosurgery 1984;15:672-8

40 McKissock W, Richardson A, Bloom WH. Subdural haematoma. A review of 389 cases. Lancet 1960;i:13659.

41 Caron JL, Worthington C, Bertrand G. Tension pneumocephalus after evacuation of chronic subdural hematoma and subsequent treatment with continuous lumbar subarachnoid infusion and craniostomy drainage. Neurosurgery 1985;16:107-10.

42 Ishiwata Y, Fujitsu K, Sekino T, et al. Subdural tension pneumocephalus following surgery for chronic subdural pneumocephalus following surgery for

43 Reinges MHT, Rübben A, Spetzger U, et al. Minimally invasive bedside craniotomy using a self-controlling presive bedside craniotomy using a self-controlling pre1998;50:226-30.

44 Reinges MHT, Rohde V, Spetzger U, et al. Modification of a mechanical twist drill trephine for craniostomy in trauma patients. Neurol Res 1999;21:108-10. 